\title{
Multivariate analysis of exploratory behavior in gerbils
}

\author{
JEFFREY ROSENFELD, LANE A. LASKO, and EDWARD C. SIMMEL \\ Miami University, Oxford, Ohio 45056
}

\begin{abstract}
Previous studies have reported that general activity can be distinguished from stimulus reactivity and is under separate genetic control in Mus musculus. The present study was designed to measure these processes in gerbils. During a 15-min test period, measures were recorded for 70 male and female gerbils at 60 and 120 days of age. The test relied more on the gerbil's tactile exploration of the stimulus and less on exploration by visual acuity from afar. A principal components factor analysis revealed two primary factors operating in the gerbils' exploratory behavior. One factor was attributed to general locomotor activity; the other was attributed to the complexity of the stimulus (stimulus reactivity). Implications on the use of this procedure for this species in future research are discussed.
\end{abstract}

A genetic model underlying exploratory behavior reported by Simmel and Eleftheriou (1977) demonstrated that two distinct factors operate in the measurement of exploratory behavior: behavior initiated by the presence of a novel stimulus (stimulus reactivity) and general activity. The measurement of exploratory behavior taking both of these factors into account provides a means for studying exploration using various species in the laboratory as well as in their natural habitats.

Naturalistic observation of the gerbil (Meriones unguiculatus) reveals the exploratory nature of the animal. Gerbils have been found to have excellent visual acuity. Thompson and Lippman (1972) indicated the importance of visual cues in the gerbil's exploratory behavior. Reliance on visual cues in exploration, however, is difficult to measure. Exploratory behavior is more commonly measured by locomotion leading to physical exploration of a novel stimulus. We conducted pilot tests, using the procedure designed by Simmel and Eleftheriou (1977), in which the activity of subjects could be measured in two compartments differing in the novelty and complexity of the visual stimuli present. The novel stimuli in those tests did not enhance physical exploration, but, as expected, the gerbils relied heavily on their visual acuity for exploration.

In order to decrease visual exploration from afar, the stimulus used must promote a greater dependence on tactile exploration. If a measure of exploratory behavior using various textured materials or tactile cues can be determined, a similar distinction between general activity and stimulus reactivity should be found. Finding this same distinction in gerbils, whose survival in natural

Request reprints from Edward C. Simmel, Behavior Genetics Laboratory, Department of Psychology, Miami University, Oxford, Ohio 45056. settings is dependent on exploration, will provide important implications for the use of gerbils in future research.

\section{METHOD}

\section{Subjects}

Forty-four male and female gerbils between the ages of 50 and 70 days were used as subjects. Twenty-six of these were tested again at 120 to 130 days of age to reveal any age-experience effects. All subjects were born and reared at Miami University's Behavior Genetics Lab from breeder stock obtained from Tumblebrook Farms, Inc., West Brookfield, Massachusetts.

\section{Apparatus}

A rectangular testing box $(87 \times 28.5 \mathrm{~cm})$ was bisected lengthwise, resulting in two halves of equal area. Each side of the arena was separated by four distinct lines (drawn on the floor) that divided the total arena into 10 squares (approximately $14 \mathrm{~cm}^{2}$ ). One side of the lateral partition contained five separate linearly arranged compartments containing textured materials. The front of the entire rectangular arena was made of clear Plexiglas, so the experimenter could observe the subjects during the entire test period. The subjects had free access to textures as well as to the plain side. Five textures were used as stimuli for the novel side: shredded newsprint (NEW); styrofoam chips (STY) used as packing material for shipping glassware; Ab-Sorb-Dri (ASD), Pell-o-cell (PEL), and San-o-cell (SAN), three bedding materials. The arena was cleaned with disinfectant after each test and new texture materials were added. The order of placement of textures remained constant from left to right: SAN, NEW, ASD, PEL, and STY. The arena was uniformly lighted from above by two $60-\mathrm{W}$ bulbs. The lights were situated approximately $15 \mathrm{~cm}$ above and were adjusted to about two-thirds of their full illumination. A radio was played during the entire test period to mask extraneous noises. An Esterline-Angus 10-channel event recorder was used for the collection and temporal sequencing of data.

\section{Procedure}

The test arena was analogous to the testing situation described by Simmel and Eleftheriou (1977). The primary difference in our study was that the stimuli used permitted tactile as well as visual exploration.

Prior to the testing period, the subjects were exposed to 
Table 1

Mean Scores (in Seconds) for the 10 Dependent Measures

\begin{tabular}{llllllllrrr}
\hline & STY & PEL & ASD & NEW & SAN & NOVT & PLNT & NOVA & PLNA & HAB \\
\hline Mean & 67.7 & 69.4 & 69.4 & 72.6 & 83.7 & 361.4 & 539.9 & 158.6 & 188.7 & 196.0 \\
\pm SE & 29.6 & 32.0 & 29.1 & 41.0 & 50.2 & 98.5 & 100.4 & 48.9 & 86.7 & 51.0 \\
\hline
\end{tabular}

Note-See text for abbreviations.

Table 2

Correlation Matrix for All Dependent Measures

\begin{tabular}{lrrrrrrrrrr}
\hline & STY & PEL & ASD & NEW & SAN & NOVT & PLNT & NOVA & PLNA & HAB \\
\hline STY & 1.00 & & & & & & & & & \\
PEL & .21 & 1.00 & & & & & & & & \\
ASD & .02 & .18 & 1.00 & & & & & & & \\
NEW & -.03 & .11 & .24 & 1.00 & & & & & & \\
SAN & .14 & .15 & -.15 & .15 & 1.00 & & & & & \\
NOVT & .39 & .52 & .39 & .63 & .60 & 1.00 & & & & \\
PLNT & -.39 & -.52 & -.38 & -.63 & -.60 & -.99 & 1.00 & & & \\
NOVA & -.02 & .15 & .15 & -.03 & -.03 & .05 & -.05 & 1.00 & & \\
PLNA & -.15 & -.07 & -.01 & -.30 & -.29 & -.35 & .34 & .68 & 1.00 & \\
HAB & -.04 & -.09 & .13 & .18 & .03 & .11 & -.10 & .33 & .23 & 1.00 \\
\hline
\end{tabular}

*Note-See text for abbreviations.

only the plain side of the box to permit habituation to that side. After habituation, when the $15-\mathrm{min}$ test period began, a removable solid partition was lifted to allow free access between the plain and novel sides.

The times spent in each of the five textures were recorded, as were the cumulative times spent in both the plain and the novel sides. The number of seconds spent on the plain side was derived from adding the times spent in each texture and subtracting from the total test time $(900 \mathrm{sec})$. The number of lines crossed on the plain and novel sides measured activity.

In summary, 10 dependent measures were obtained from this procedure: (1) STY: time spent (in seconds) exploring styrofoam. (2) PEL: time spent (in seconds) exploring Pel-ocell. (3) ASD: time spent (in seconds) exploring Ab-Sorb-Dri. (4) NEW: time spent (in seconds) exploring newsprint. (5) SAN: time spent (in seconds) exploring San-o-cell. (6) Plain side time (PLNT): time spent on the plain half of the arena. (7) Novel side time (NOVT): time spent on the novel half of the arena. (8) Plain activity (PLNA): frequency of lines crossed on the plain side. (9) Novel activity (NOVA): frequency of lines crossed on the novel side. (10) Habituation (HAB): frequency of lines crossed on the plain side during the habituation period.

\section{RESULTS AND DISCUSSION}

The novelty in the test arena was composed of bedding materials of five different textures. The time spent exploring or reacting to the novel setting is defined as stimulus reactivity. The stimuli were analyzed to determine if the various textures contributed evenly to the stimulus reactivity of the subject. Means were computed for time spent in each texture (see Table 1) and a series of $t$ tests were computed comparing the mean time spent in each texture; no significant differences were found. All the textures, then, contributed to the novelty effect. Pettijohn and Barkes (1978) have reported significant preferences to certain textures. In that study several similar textures were used; however, in our study we found no significant preference to any one texture.
A principal-components factor analysis (SPSS; 1975) was performed on the data from the 10 dependent measures based on the correlation matrix shown in Table 2. The factor loadings on these measures following a Varimax rotation are shown in Table 3.

Two of our measures (PLNA and NOVA) were designed to record general activity, while seven measures (STY, PEL, ASD, NEW, SAN, NOVT, and PLNT) were designed to record stimulus reactivity as defined by Simmel and Eleftheriou (1977). The activity measures had high loadings only on Factor 2 , while all the other measures had low loadings on this factor (see Table 3). The distinction between stimulus reactivity and general activity established by this factor structure was consistent with that found for inbred mice by Simmel and Eleftheriou.

The stimulus reactivity measures had high loadings spread over the remaining three factors. The reasons these measures comprised three factors instead of one, a stimulus reactivity measure, can be attributed to at least two sources. Firstly, a large proportion of the variance was due to individual differences which emerged as random sources of error. This source of

Table 3

Factor Loadings Following a Varimax Rotation

\begin{tabular}{lrrrr}
\hline & 1 & \multicolumn{1}{c}{2} & \multicolumn{1}{c}{3} & \multicolumn{1}{c}{4} \\
\hline STY & .038 & -.068 & .678 & -.150 \\
PEL & .195 & .079 & .751 & .158 \\
ASD & .413 & .114 & .154 & .755 \\
NEW & .838 & -.101 & -.120 & .154 \\
SAN & .489 & -.033 & .263 & -.715 \\
NOVT & .845 & -.027 & .519 & -.061 \\
PLNT & -.834 & .027 & -.527 & .064 \\
NOVA & .008 & .904 & .148 & .073 \\
PLNA & -.383 & .821 & -.053 & .116 \\
HAB & .397 & .590 & -.352 & -.089
\end{tabular}


variation comprised excess factors that might appear incorrectly as distinct experimental effects. We found through a previous factor structure earlier in the study that, as sample size increased (tested at $\mathrm{N}=53$ ), the number of factors presumably due to individual difference decreased. Second, two of the measures considered in the seven associated with stimulus reactivity, NOVT and PLNT, were derived as a cumulative result of the other five. These measures result from interdependent data: Any sources contributing to the variance of the five component measures would also directly affect NOVT and PLNT. The factor structure associated the two measures on a separate factor. Since these data are actually composed of five component measures and their sum, it cannot be assumed that the component measures account for an experimental effect independent of their sum. The data, then, do support only two experimental effects, one associated with general activity and one associated with stimulus reactivity.

The distinction in exploratory behavior as discussed affords many opportunities for future research with gerbils. Using inbred strains of mice, this behavioral distinction was traced to two genetic models controlling both aspects of exploratory behavior (Simmel \& Eleftheriou, 1977). Our study introduced a reliable test through which such genetic research could be initiated with gerbils. Understanding the mechanism controlling a naturalistic response such as exploratory behavior can serve an instrumental function in developmental studies. Using this test as a dependent measure, studies of early experience, drug manipulation, or aging become possible. There were some preliminary indications in the group retested at 120 days that both activity and reactivity scores decreased as a function of age.

Throughout the course of this study, stress responses were also elicited by the stimuli we used. Some subjects reacted to the test arena by going into seizure-like behavior. Usually these seizures lasted a maximum of 3 min and, while exploration was certainly limited, clear indication was given that the animal was stressed. This fairly low threshold to stress suggests that the gerbil could serve as a prime subject for physiological studies on the effects of such stimuli.

\section{REFERENCES}

Pettijohn, T. F., \& Barkes, B. M. Surface choice and behavior in adult Mongolian gerbils. Psychological Record, 1978, 28, 299-303.

Simmel, E. C., \& Eleftheriou, B. E. Multivariate and behavior genetic analysis of avoidance of complex visual stimuli and activity in recombinant inbred strains of mice. Behavior Genetics, 1977, 7, 239-250.

Thompson, R. W., \& Lippman, L. G. Exploration and activity in the gerbil and the rat. Journal of Comparative and Physiological Psychology, 1972, 80, 439-448.

(Received for publication June 30, 1978.) 